\title{
Aortic aneurysm in a patient with long-standing hypothyroidism
}

\author{
Caroline Savage \\ M.B., B.S., M.R.C.P.
}

\author{
J. E. DEANFIELD \\ M.B., B.Ch., M.R.C.P.
}

\author{
R. T. JUNG \\ M.A., M.D., M.R.C.P. \\ Hammersmith Hospital, Ducane Road, London W12 0HS
}

\begin{abstract}
Summary
Hypothyroidism over a lifetime is rare. Such a case is reported due to post-coupling dyshormonogenesis in a patient who had developed an aortic aneurysm. It is proposed that the aortic aneurysm could have arisen as a direct consequence of the hypothyroidism. Furthermore, Reilly bodies, which are deposits of mucopolysaccharide, were found in the peripheral blood leucocytes; these have never been previously described in hypothyroidism. It is suggested that they may reflect an underlying disturbance in mucopolysaccharide turnover which could have resulted in weakening of the aortic wall and aneurysmal formation
\end{abstract}

\section{Introduction}

There is a recognized disturbance in mucopolysaccharide synthesis and degradation in hypothyroidism (Schiller, Slover and Dorfman, 1962). It is known that this disturbance may effect the mucopolysaccharide content of the human aorta (Jo'zsa and Szederke'nyi, 1966). However, no direct pathological effects of this have yet been described. Reilly bodies were first described in the primary mucopolysaccharide disorders (Reilly, 1941), and have been considered specific to these conditions. It seems possible that they could simply reflect a disordered mucopolysaccharide turnover, regardless of how this arises, including the altered mucopolysaccharide handling found in hypothyroidism.

\section{Case report}

A 52-year-old man presented with a 10 year history of intermittent retrosternal stabbing chest pains, unrelated to exertion. He had also noticed increasing deafness, increasing cold intolerance and tiredness over 3 years. He had been treated for hypothyroidism at the age of 5 years, but had discontinued therapy at
14 years for unknown reasons. He had a sister and younger twin brothers who were known to suffer from hypothyroidism, secondary to a post-coupling defect in thyroid hormonogenesis with the release of an abnormal iodoprotein. He was a short and stocky man, with a waxen facies and generally slow in his movements. He had a very gruff voice. A soft diffuse goitre extended retrosternally with no evidence of tracheal obstruction. His skin was not dry and reflexes showed a normal relaxation time. He was in sinus rhythm with a collapsing pulse and a blood pressure of $160 / 80 \mathrm{~mm} \mathrm{Hg}$. Auscultatory signs were consistent with moderate aortic incompetence. $\mathrm{He}$ had bilateral neural deafness but the rest of the examination was normal. Investigations confirmed a diagnosis of hypothyroidism with a serum thyroxine of less than $10 \mathrm{nmol} /$ litre (normal range 71-148 nmol/litre), serum triiodothyronine of less than 1 nmol/litre (normal range 1.5-3.1 nmol/litre) and a thyroid-stimulating hormone level of greater than 25 mu./litre (normal range $<4.6 \mathrm{mu}$./litre). The free thyroxine index was 8.5 (normal range 45-160). In view of the family history a dyshormonogenic cause for his hypothyroidism was sought. The technetium$99\left({ }^{99} \mathrm{Tc}\right)$ uptake was elevated at $3.6 \%$ at 20 mins after injection (normal range $(0.4-3 \%)$, and there was no discharge with perchlorate after $100 \mathrm{mCi}$ injection of ${ }^{131}$ I. These results indicated that his hypothyroidism was due to dyshormonogenesis at coupling or beyond. Butanol extractability to detect an abnormal iodoprotein could not be performed due to inadequate counts in the peripheral blood. The other important finding was that of a dilated ascending aorta seen on the chest X-ray. Angiography confirmed the presence of a fusiform aneurysmal dilatation of the ascending aorta, which was $7.2 \mathrm{~cm}$ wide at the aortic root, and moderate aortic regurgitation; the distal transverse and descending aorta were normal. There was no evidence of an underlying rheumatological disorder as the erythrocyte 
sedimentation rate was normal and the latex test and anti-nuclear factor tests were negative. Specific serology for syphilis was negative. Objective evidence of myocardial ischaemia was sought to explain his chest pain but the electrocardiogram during pain, an exercise electrocardiogram, and 24-hr ambulatory ST segment monitoring were all negative.

Surprisingly, a peripheral blood film revealed deposits in the leucocytes which were confirmed to be deposits of mucopolysaccharide using a GiemsaWright stain. These inclusions are known as Reilly bodies, normally specific to the primary mucopolysaccharide disorders (Reilly, 1941). Urinary glycosaminoglycans and chromatography excluded the presence of an underlying mucopolysaccharide disorder. No deposits of mucopolysaccharide were seen in the bone marrow leucocytes. Bone X-rays showed no evidence to support a mucopolysaccharide disorder and there were no corneal opacities on slit lamp examination.

The hypothyroidism was treated initially with thyroxine $25 \mu \mathrm{g}$ per day and gradually increased to $150 \mu \mathrm{g}$ per day. On this treatment the patient's appearance improved; he became more active, his chest pain disappeared and the Reilly bodies were no longer seen in the peripheral blood film.

\section{Discussion}

This patient is unusual in so far as he managed to sustain a hypothyroid state for nearly $\mathbf{4 0}$ years. The elevation of ${ }^{99} \mathrm{Tc}$ uptake and negative perchlorate discharge tests excluded an iodine trapping or an organification defect respectively. We were unable to show whether he had a coupling or post-coupling defect but comparison with the investigations done on his three siblings suggest that he too probably had a post-coupling dyshormonogenesis. It seems likely that he was producing some thyroxine, albeit reduced amounts, to explain his prolonged survival and the incomplete clinical picture with which he finally presented.

There is circumstantial evidence that the aortic aneurysm was more than an incidental finding. Studies on post-mortem samples of human aorta in hypothyroidism show an absolute increase in mucopolysaccharide content, with an increase of hyaluronic acid and a decrease of chondroitin sulphate, (Jo'zsa and Szederke'nyi, 1966). Dorfman has suggested that this altered mucopolysaccharide handling in hypothyroidism is due to a decrease in catabolism of hyaluronic acid and a decrease in synthesis of chondroitin sulphate (Dorfman, 1963). The finding of Reilly bodies in this patient which disappeared following treatment with thyroxine suggests that abnormal mucopolysaccharide turnover was indeed present. Reilly bodies have not been described in hypothyroidism before but may reflect the abnormal mucopolysaccharide handling in this condition. If the mucopolysaccharide content of the aorta was also affected (as in the post-mortem studies), this may have caused weakening of the wall and allowed the development of an aneurysm, with secondary aortic valve incompetence. The long duration of the hypothyroid state may have been necessary to allow all these secondary effects to occur. Furthermore, it is unusual for an aortic aneurysm to develop in a relatively young man, in the absence of syphilis or an associated rheumatological disorder, strengthening the suggestion of a link with the hypothyroid state.

\section{Acknowledgments}

We thank Professor J. F. Goodwin for allowing us to report the case and Dr G. F. Joplin for his advice.

\section{References}

DORFMAN, A.J. (1963) Polysaccharide of corrective tissue. Journal of Histochemistry \& Cytochemistry, II, 2.

Jo'ZSA, L. \& SZEDERKE'NYI, J. (1966) The mucopolysaccharide content of the human aorta in hypothyroidism. Endocrinologie, $\mathbf{5 0}$, 116.

REILLY, W.A. (1941) The granules in the leucocytes in gargoylism. American Journal of Diseases of Children, 62, 489.

SCHILler, S., Slover, G.A. \& DORFMAN, A.J. (1962) Effect of the thyroid gland on metabolism of acid mucopolysaccharide in skin. Biochimica et biophysica Acta, 58, 27. 